\title{
EDITORIAL
}

Check for updates

\section{Three years, in a network}

December 2021 closes our third year of publication. To celebrate, we look at the links between our review-type articles, and think about what articles we might publish next.

The nodes in the network represent the Reviews, Technical Reviews, Perspectives, Expert Recommendations and Roadmaps we have published since launching in

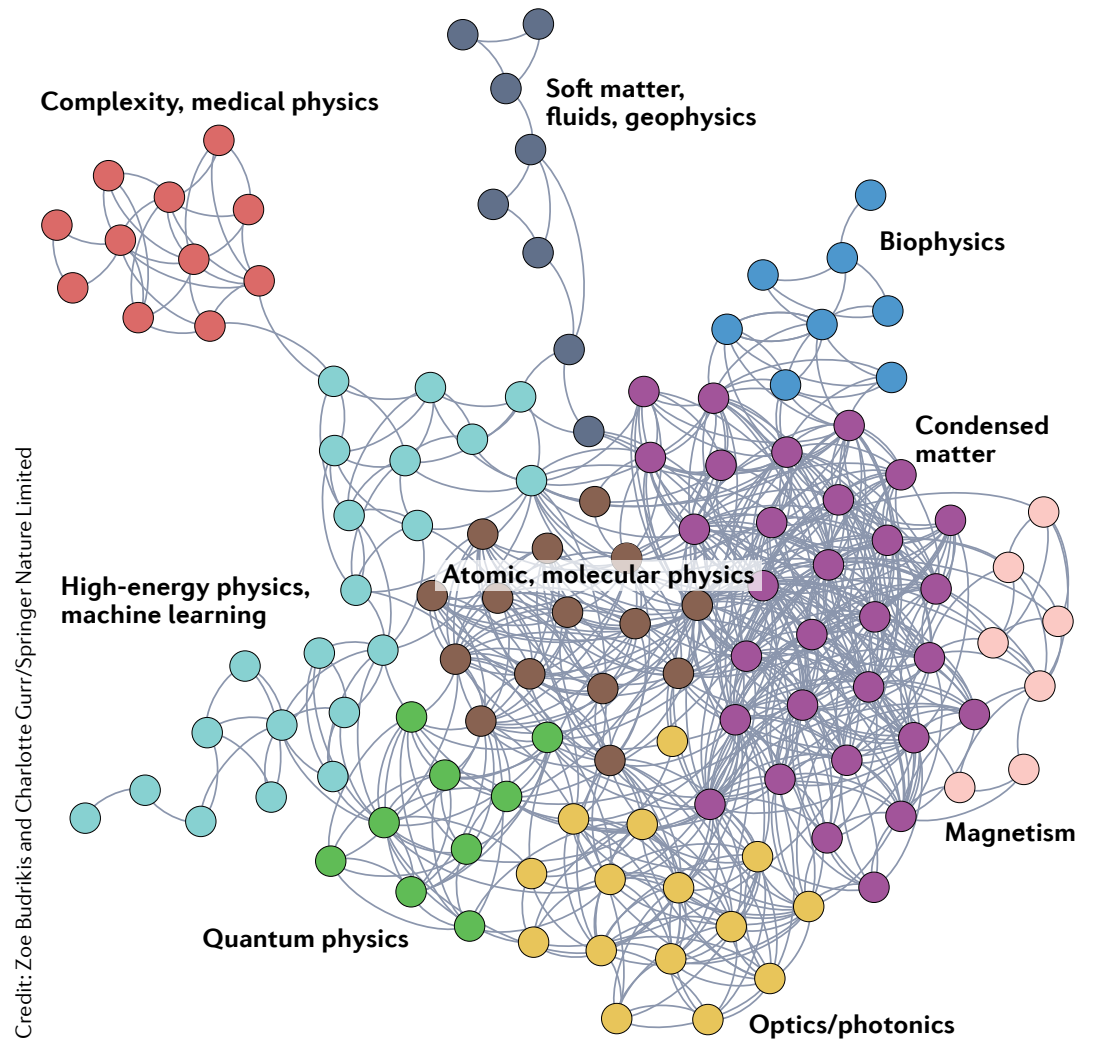

January 2019. We formed the network by associating with each article a list of keywords from the PhySH ontology ${ }^{1}$, which could be research areas, physical systems or techniques; articles are linked on the network if they share at least one keyword.

The colours of the nodes represent the communities that were detected on the network, that is, groups of nodes for which the links within the group are tighter than the links between groups. The community detection algorithm ${ }^{2}$ takes into account only the network links, without any information about the content of the articles, but the communities align quite well with subject areas. (In the figure we have labelled the communities based on our own perception of what topics they cover.) There are some notable groupings of subject areas, such as the community that takes in both high-energy physics and machine learning-based articles, which form one community thanks to a number of articles that employ machine learning techniques in high-energy physics.

One piece of information we can learn from the network is which pairs of communities have no direct links - the missing link would be an interdisciplinary article that unites topics and/or techniques from two different subfields of physics. We look forward to commissioning such articles in the years to come.

1. Smith, A. From PACS to PhySH. Nat. Rev. Phys. 1, 8-11 (2019).

2. Blondel, V. D. et al. Fast unfolding of communities in large networks. J. Stat. Mech. 2008, P10008 (2008). 